\title{
LA DESCOSIFICACIÓN DE LOS ANIMALES
}

\author{
THE DE-OBJECTIFICATION OF ANIMALS
}

\begin{abstract}
MARITA GIMÉNEZ-CANDELA
Catedrática de Derecho Romano en la Universitat Autònoma de Barcelona. Directora del Máster en Derecho Animal y Sociedad. Directora del ICALP. Fundadora y Editora de www.derechoanimal.info maritacandela@hotmail.com
\end{abstract}

\section{RESUMEN}

La cuestión de la propiedad sobre los animales, ha sido pacífica e indiscutida hasta épocas muy recientes. La Descosificación de los animales, que se propone ahora en el Código civil de España, se ha llevado a cabo recientemente en los Códigos civiles de Francia (2015) y Portugal (2016) y se está perfilando como un movimiento que, con intermitencias, no ha cesado de avanzar desde que en 1988 en Austria se introdujera la afirmación de que los animales no son cosas ("nichtSachen"). Hoy Descosificación se equipara a reconocimiento de la sentiencia animal ("sentient beings").

Palabras-clave: Descosificación de los animales; estatuto jurídico de los animales; seres sintientes; derecho Animal; Codigo Civil.

\begin{abstract}
The question about the property over animals has been peaceful and undisputed until very recent times. The de-objectification of animals, which is now proposed in the Civil Code of Spain, has recently been implemented in the Civil Codes of France (2015) and Portugal (2016) and is emerging as a movement that, with intermittency, does not has ceased to move forward since the introduction in Austria in 1988 that animals are not things of property ("nicht-Sachen"). Today De-objectification is equated with recognition of the animal sentience ("sentient beings").
\end{abstract}

Keywords: De-objectification of animals; Civil law; legal status of animals; animal sentience; Animal Law. 


\section{SUMÁRIO}

1 PERSPECTIVA HISTÓRICA; 1.1 El mundo romano; 1.2 La Codificación; 1.3 Una cuestión global; 2 LA SENTIENCIA ANIMAL; 2.1 El planteamiento; 2.2 La noción de sentiencia animal; 2.3 La aplicación normativa; 2.4 Qué significa Descosificación de los animales.

\section{PERSPECTIVA HISTÓRICA}

\subsection{El mundo romano}

Los animales han jugado un papel de gran importancia dentro de la sociedad, el derecho y la economía a lo largo de toda nuestra trayectoria histórica. A pesar de que se haya olvidado muchas veces el universal respeto por los animales, que es característico del Mundo Antiguo y que transmite el jurista Ulpiano, en el famoso texto del Digesto D. 1,1,1,3 ${ }^{1}$, en el que refiere la existencia y aplicación de un derecho propio de la naturaleza que es común a todos los seres vivos (sin distinción de si se trata de humanos o animales), toda nuestra cultura está impregnada por un profundo conocimiento y persuasión colectiva de que los animales son criaturas que comparten nuestro destino, lo que de modo sintético y eficaz proclama la expresión alemana “Mitgeschöpfte", para referirse a los animales ${ }^{2}$. Esta posición, remite fundamentalmente al respeto y veneración del hombre por la naturaleza que, en nuestro mundo occidental ${ }^{3}$, se ha ido desdibujando con el paso de los siglos, pero que es el que nos ha transmitido toda la literatura clásica greco-romana ${ }^{4}$.

Los animales tienen en nuestro sistema jurídico continental, la consideración de cosas en propiedad. La atribución del estatuto de cosas $(r e s)^{5}$, referido a los animales, es una creación de la técnica jurídica romana, que, en su momento, integra a las dos principales fuerzas de trabajo de una sociedad eminentemente agrícola (los esclavos y los animales, sobre todo los de tiro y carga) dentro de los elementos más importantes del patrimonio (mancipium) del jefe de

\footnotetext{
${ }^{1}$ ULPIAN D. 1,1,1,3: lus naturale est, quod natura omnia animalia docuit: nam ius istud non humani generis proprium, sed omnium animalium, quae in terra, quae in mari nascuntur, avium quoque commune est. hinc descendit maris atque feminae coniunctio, quam nos matrimonium appellamus, hinc liberorum procreatio, hinc educatio: videmus etenim cetera quoque animalia, feras etiam istius iuris peritia censeri.

${ }^{2}$ OBERGFELL, E.I., Tiere als Mitgeschöpft im Zivilrecht, en Rechtswissenschaft 3 (2016) 388ss.

${ }^{3}$ KELCH, T., A Short History of (mostly) Western Animal Law: Part I, Animal Law Review (2012) 1ss.; Part II, Animal Law Review (2013) 1ss.

${ }^{4}$ Vid. Entre otros, DIERAUER, U., Tier und Mensch im Denken der Antike (Amsterdam 1977); INGVILD GILHUS, A., Animals, Gods and Humans (London-New York 2006); ALEXANDRIDIS, WILD, WINKLER-HORACEK (ed.), Mensch und Tier in der Antike (Wiesbaden 2008).

${ }^{5}$ GIMÉNEZ-CANDELA, T., Derecho Privado Romano (Valencia 1999)
} 
un clan familiar (paterfamilias), es decir, del dueño y único titular del conjunto de los bienes con los que una familia desenvolvía su vida y su actividad económica.

En su momento, la inclusión de los animales dentro del sistema jurídico romano, como una categoría específica dentro de los elementos integrantes de la propiedad, significó un cambio muy importante, pues en otras sociedades de la Antigüedad, los animales y los esclavos existían (por supuesto!), pero fuera de la realidad jurídica, es decir, no incluidos dentro de un sistema de Derecho. De ahí que, como es bien sabido, otorgar a los esclavos y a los animales el mismo estatuto jurídico, por mucho que hoy nos parezca rechazable y paradójico, es lo que ha permitido que la situación de los esclavos, en el devenir histórico, mejorara a través de la manumisión y, definitivamente se aboliera. La abolición de la esclavitud no hubiera sido posible de no haber figurado los esclavos como una categoría jurídica. En aplicación de la misma lógica, hoy en día nos planteamos el cambio de estatuto jurídico de los animales, como cosas en propiedad, porque están identificados como una categoría jurídica y éstas admiten cambios, mejoras y supresiones.

Que en una sociedad configurada como la que sumariamente he descrito, los animales (básicamente, los que servían para el transporte, el sustento y el trabajo) ocuparan durante siglos un lugar indiscutido dentro de la propiedad, se justifica principalmente porque la sociedad no cambió sustancialmente de forma y estructura hasta muy recientes siglos, particularmente, a partir de la Revolución Industrial. Por lo tanto, el Derecho, conforme a un panorama social y económico muy semejante al de la Antigüedad clásica, no se preocupó de introducir cambios -ni siquiera de planteárselos-respecto a la relación jurídica con los animales. La cuestión de la propiedad sobre los animales, ha sido pacífica e indiscutida hasta épocas muy recientes ${ }^{6}$.

\subsection{La Codificación}

Todo el legado del Derecho Romano se vertió, de modo prácticamente inalterado, a través de la Codificación napoleónica al mundo contemporáneo. No es de extrañar que el propio Napoleón (“...un nuevo Derecho para un nuevo ciudadano”) insistiera con lucidez -aún en medio de la penuria de sus últimos años de destierro en un islote azotado por el viento-, en que su nombre no aparecería asociado para la posteridad, a los de las grandes batallas con las que se

\footnotetext{
${ }^{6}$ MUÑOZ MACHADO, S., Los animales y el Derecho (Madrid 1999) 70s.
} 
había adueñado de Europa, sino al de la recopilación unificada del Derecho que él encargó llevar a término a ilustres juristas y que aún hoy se conoce como Code civil o Code Napoleon ${ }^{7}$.

El Derecho, a través de la Codificación, se ha ocupado de los animales -dentro de la lógica de la pertenencia a las cosas-, en la medida en que aquéllos han cubierto las necesidades más elementales de la vida: como productos, como medios de transporte, como medios para la investigación, como compañía, como parte de los espectáculos dirigidos a divertir al ser humano. Así mismo, el Derecho se ha ocupado de los animales en la medida en que han sido fuente de responsabilidad, en caso de conflictos de intereses, mediando o participando un animal en tal conflicto.

Cuestión aparte, en mi opinión, es la respuesta que el Derecho ha dado al maltrato animal a través del Derecho Penal, pues es misión de un Estado constitucional democrático proveer de sanciones, frente los comportamientos que cuestionan la eficacia de la facultad punitiva que le incumbe ${ }^{8}$. Éste es un mandato, en el que, naturalmente la cuestión de la propiedad sobre los animales juega también un papel ${ }^{9}$, pero el castigo por el maltrato animal se asocia a los comportamientos que la ley determina como ilícitos y en los que el Estado asume una particular misión de vigilancia frente a los más vulnerables ${ }^{10}$.Sin embargo, el pensamiento crítico sí ha recogido en muchas ocasiones a lo largo de los siglos, la inicial consideración de respeto universal por los animales y el deseo de protegerlos frente a los abusos y agresiones, para suscitar la cuestión de atribuirles una posición jurídica mejor que la de la propiedad sobre ellos. Dicho en otros términos, la Filosofía ${ }^{11}$, la Ética ${ }^{12}$, la Antropología, la Historia, y recientemente la Ciencia del Bienestar Animal, sí se han cuestionado que los animales permanezcan ligados al estatuto de cosas en propiedad y, además, que dicho estatuto justifique,

\footnotetext{
${ }^{7}$ Llamado "Le Code civil des Français", se promulgó el 21 de marzo de 1804 y empezó a conocerse como Code Napoleon desde 1807.

8 RIOS CORBACHO, JM., Comentario en relación al maltrato de animales. L01/2015 (http://www.derechoanimal.info/images/pdf/Corbacho-Reforma.pdf; REQUEJO CONDE, MC., El delito de maltrato a los animales tras la reforma del Código Penalpor la ley Orgánica 1/2015 de 30 de marzo (http://www.derechoanimal.info/images/pdf/Requejo.pdf)

9 WOHLERS, W., Tierschutz durch Strafrecht?. Zur Legitimation tierschutzstrafrechtlicher Normen, en Rechtswissenschaft 3 (2016) 426ss.

${ }^{10}$ ROXIN, K., Sinn und Grenzen Staatlicher Strafe, JuS (1996) 377, 383n.20: “...im Schmerzempfinden der Tiere, dem sich die Rechtsordnung aus einer Art von kreatürlicher Solidarität annimmt".

${ }_{11}$ REGAN, T., The Case for Animal Rights (University California Press 1983); CAPACETE, F., En recuerdo de Tom Regan(http://www.derechoanimal.info/esp/page/5091/en-recuerdo-de-tom-regan)

${ }^{12}$ POLLO, S., Umani e Animali: questioni di Etica (Roma 2016).
} 
no ya el uso sobre ellos, sino también el abuso sin límites sobre la vida y la integridad física de los animales ${ }^{13}$.

Por lo dicho, queda patente que, a día de hoy, la cuestión del estatuto jurídico de los animales y su eventual cambio, concretamente en el Código civil, ha dejado de ser una cuestión que sólo interesa y moviliza a los movimientos de defensa de los animales (que también!), para pasar a ser una cuestión que:

-preocupa a la Ciencia y al lenguaje jurídico acerca de los animales como seres sintientes ("sentient beings")

-preocupa a la sociedad en su conjunto, que ha desarrollado una nueva sensibilidad -preocupa a la organización política del Estado, que asume su responsabilidad frente al Bienestar Animal que es uno de los principios rectores de la UE

-preocupa a la economía y a la educación en el respeto a los animales, en todos los niveles de la enseñanza.

\subsection{Una cuestión global}

Que la cuestión animal es una cuestión global, se evidencia con sólo echar una mirada a la evolución del Derecho Animal ${ }^{14}$, una disciplina emergente del Derecho, que se afirma, cada vez más como un instrumento que facilita el "giro" que precisa el Derecho para abrirse a nuevas perspectivas y admisión de nuevas fronteras ${ }^{15}$, que, a mi modo de ver se pueden resumir en:

Descosificación de los animales, Constitucionalización de los animales y Globalización de los animales.

Sobre Descosificación de los animales vengo insistiendo desde mis primeros escritos en tema de Derecho Animal y figura como hilo conductor de todas las investigaciones y proyectos que desde la web derechoanimal.info (http://www.derechoanimal.info/esp/page/1434/introduccion) y desde el ICALP se han alentado.

\footnotetext{
${ }^{13}$ BOISSEAU-SOWINSKI, L., La désappropiation de l'animal (Presses Universitaires de Limoges 2013).

${ }^{14}$ Por todos, FAVRE, D., Animal Law, Welfare, Interests and Rights (New York 2011); How Common Law Equity Concepts can help enhance Animal's Status, en Animals and the Law (Valencia 2015)181ss.

${ }^{15}$ PETERS, A., Vom Tierschutzrecht zu Legal Animal Studies: Forschungsdesiderate und -perspectiven, en 3 (2016)332s.
} 
Que el Código civil español reconozca a los animales como "seres vivos dotados de sensibilidad", al igual que han hecho los Códigos civiles de Francia (2015) ${ }^{16}$ y de Portugal $(2016)^{17}$, no sería más que el resultado de una actuación consecuente del Gobierno a quien una reciente petición de reforma, iniciada por el "Observatorio de Justicia y Defensa Animal” y avalada por más de un cuarto de millón de firmas de ciudadanos $^{18}$, ha instado a iniciar los trámites para "promover las reformas legales necesarias para crear una categoría especial en el Código civil distinta a las ya previstas, referida a los animales, donde se les defina como seres vivos dotados de sensibilidad" ${ }^{19}$.

Antes de las reformas llevadas a cabo en Francia y Portugal, la "Descosificación" de los animales se ha perfilado como un movimiento que, con intermitencias, no ha cesado de avanzar desde que en 1988 en Austria se introdujera, en la clasificación de los animales, la afirmación, formulada de forma negativa, de que los animales no son cosas ("nicht-Sachen") ${ }^{20}$. Esta misma formulación (“nicht-Sachen") de que los animales son “no-cosas”, es la que se siguió en Alemania, Suiza, Liechtenstein en los siguientes años ${ }^{21}$, con diferentes resultados en cada uno de estos países.

Cabe decir, también, que aunque no se haya logrado -tampoco se pretendía- que los animales sean equiparados a los seres humanos en cuanto a prerrogativas y consecución de "derechos subjetivos", sí cabe destacar que la modificación del estatuto jurídico de los animales

${ }^{16}$ Entre sus muchos escritos sobre el tema, vid. esp.: MARGUÉNAUD, JP., L'entrée en vigueur de "l'amendement Glavany": un grand pas de plus vers la personnalité juridique des animaux, en RSDA 2 (2014) 15ss.; GIMÉNEZ-CANDELA, T., Una nueva Revolución Francesa: la modernización del Code civil (http://www.derechoanimal.info/esp/page/3718/una-nueva-revolucion-francesa-la-modernizacion-delcode-civil

17 ARAUJO, F., A hora dos Direitos dos Animais. O Obstaculo da Apropriaçao (Coimbra 2003) 303ss.; GIMÉNEZ-CANDELA, T., Reforma del Cc. de Portugal: los animales como seres sintientes (http://www.derechoanimal.info/esp/page/4990/reforma-del-cc-de-portugal-los-animales-como-seressintientes)

${ }^{18}$ Codina, Jl., Unanimidad en el Congreso de los Diputados para instar la reforma del Código civil Español y reconocer a los animales como seres dotados de sensibilidad (http://www.derechoanimal.info/images/pdf/SeresDotadosSensibilidad.pdf

19 Diario de Sesiones del Congreso de los Diputados Pleno y Diputación Permanente, 14.2.2017 43ss.(http://www.congreso.es/public_oficiales/L12/CONG/DS/PL/DSCD-12-PL-29.PDF)

${ }^{20}$ ABGB §285a, de 1 de Julio de 1988 (BGBI 179/188; JGS Nr.946/1811):“ § 285a Tiere sind keine Sachen; sie werden durch besondere Gesetze geschützt. Die für Sachen geltenden Vorschriften sind auf Tiere nur insoweit anzuwenden, als keine abweichenden Regelungen bestehen. $(=$ Animals are not things; they are protected by special statutes. Provisions applicable to things only apply to animals to the extent there are no different provisions).

${ }^{21}$ Alemania: BGB $\$ 90^{\mathrm{a}}$, de 20 de Agosto de 1990, BGBI I, p.1762); Suiza: ZGB, modificación de 4 de Octubre de 2002 (AS 2003, 463; BBI 2002 4164, 5806); Liechtenstein: Art. 20a Liechtensteinisches Sachenrecht, de 14 de Mayo de 2003, sobre el cambio del Derecho de Propiedad, LR 214.0, 2003 N. 155) 
está reforzando en todos estos países el ámbito de aplicación jurídica de la condición de los animales como lo que son: seres sintientes.

Sobre los concretos significados y extensión de esta expresión, procedente de la Ciencia del Bienestar Animal y de su integración en el ámbito jurídico, me ocuparéa continuación.

\section{LA SENTIENCIA ANIMAL}

\subsection{El planteamiento}

Los Códigos civiles europeos, de forma mayoritaria, coinciden en la calificación jurídica de los animales como cosas en propiedad (res), en razón de la tradición romana a la que hemos hecho referencia ${ }^{22}$, que impregna el Derecho Privado Europeo, como fundamento histórico del mismo. En los Estados miembros de la UE, se han venido integrando muchas directivas europeas y se han ratificado, así mismo, convenciones del Consejo Europeo, que, de una forma cada vez más explícita, han favorecido que en todos los países de nuestro entorno, se haya desarrollado una decidida legislación proteccionista respecto a los animales ${ }^{23}$, inspirada por la difusión, cada vez más amplia -y recogida por el propio Tratado de Lisboa, la llamada Constitución Europea ${ }^{24}$ de que los animales son "seres sentientes" (sentient beings) y, por tanto, su tratamiento y su regulación por el Derecho debe ser acorde con tal realidad.

La resultante es un régimen jurídico, cuya primacía sigue siendo ostentada por el estatuto que a los animales les otorgue el Código civil nacional, fenómeno, como es bien sabido, dependiente de las propias raíces de la Codificación Europea y Latinoamericana, pero que, en razón de la legislación europea de Bienestar animal y de la progresiva transformación de las actitudes de la sociedad respecto a los animales, ha dado como resultado que los Códigos civiles de muchos países europeos (y de fuera del marco europeo, como Nueva Zelanda, Canadá, o Colombia) hayan cambiado el estatuto jurídico de los animales de cosas a "seres sentientes".

La cuestión que conviene plantearse, es la de la oportunidad de introducir ahora un cambio respecto al estatuto jurídico de los animales en nuestro Código civil -la "Descosificación de los mismos-, tal y como la sociedad, a través del Congreso de los Diputados ha refrendado de

\footnotetext{
${ }^{22}$ GIMÉNEZ-CANDELA, T., La Descosificación de los Animales (http://www.derechoanimal.info/esp/page/5108/la-descosificacion-de-los-animales-(I))

${ }^{23}$ N. Pedersen, European Animal Welfare Laws: Past, Present and Future, en Animal Law, 2009 (https://www.animallaw.info/intro/eu-us-comparative-cruelty-laws-2003-present)

${ }^{24}$ TFUE, en http://noticias.juridicas.com/base_datos/Anterior/r3-ttce.html
} 
forma unánime ${ }^{25}$. Es claro que la Descosificación de los animales es un movimiento que parece haber llegado para quedarse. No se trata de una moda o de una tendencia ${ }^{26}$, sino del despertar de una nueva conciencia jurídica sobre el trato que debe dispensar a los animales una sociedad del S.XXI ${ }^{27}$. En este, sentido, los primeros pasos, se dieron en el Reino Unido ${ }^{28}$.

La sentiencia animal, sin embargo, no resulta tan obvia para el legislador y quizá valga la pena detenerse a observar:

-qué implicaciones tiene,

-cómo se ha introducido hasta ahora en los textos jurídicos y

-qué cabe esperar de una adecuada colaboración entre científicos y juristas.

Dicho de otro modo:

-cuando hablamos de reconocer jurídicamente a los animales como seres sentientes, qué queremos decir?, qué consecuencias aplicables a los textos legales debemos plantearnos los juristas?,

-cómo debemos reflejar en los textos jurídicos la capacidad de respuesta de los seres humanos respecto a los animales, si los reconocemos como seres que "sienten" del mismo modo que nosotros?,

-qué resultados concretos debemos plantear y exigir, en términos de responsabilidad, tanto pública como privada, de la afirmación en los textos legales de la sentiencia animal?.

\subsection{La noción de sentiencia animal}

Un breve repaso a cómo se ha introducido la noción de sentiencia animal, como estándar de trato a los animales, arroja como resultado que la percepción de que los animales sienten es muy antigua y sí que ha influido en dispensar un trato de respeto a los animales ${ }^{29}$, sin embargo no se afirma como un concepto aceptado por la ciencia más que paralelamente al

\footnotetext{
${ }_{26}^{25}$ Vid supra, n. 18.

Hall, B.-Arellano, S.-Ramírez Barreto, C., En memoria de Tom Regan (http://www.derechoanimal.info/esp/page/5109/en-memoria-de-tom-regan)

27 Sobre este aspecto, vid. GIMÉNEZ-CANDELA, T., An Overview of Spanish Animal Law, en FAVRE, D. y GIMÉNEZ-CANDELA, T. (Ed.), Animales y Derecho (Valencia 2015) 211ss.

28 GIMÉNEZ-CANDELA, T., Brexit y los animales. El legado del Reino Unido al Derecho Animal (http://www.derechoanimal.info/esp/page/4752/brexit-y-los-animales-el-legado-del-reino-unido-alderecho-animal)

${ }^{29}$ DUNCAN, I.J.H., The Changing Concept of Animal Sentience, en Applied Animal Behaviour Science 100 (2006) 11 ss.
} 
crecimiento de la propia Ciencia del Bienestar Animal, que, como tal especialidad veterinaria, no aparece hasta el S.XX.

En el ámbito legal, la UE debe al Reino Unido algo tan importante como la creación del término científico "Bienestar Animal" (Animal Welfare) ${ }^{30}$, la forma de aplicación del mismo a través de las llamadas cinco libertades ("Five Freedoms") por las que debería regirse la regulación de los contenidos mínimos de la vida de los animales y, en las últimas décadas, la inclusión del término "sentient beings" (seres sentientes) como estándar de tratamiento de los animales, a los que se les reconoce su capacidad no sólo de experimentar dolor físico, sino sufrimiento, pero también placer y diversión. Es decir, la legislación de los últimos 40 años de la UE respecto de los animales (producción, experimentación, transporte, espectáculos) ${ }^{31}$, no se entendería probablemente sin la decisiva intervención y creatividad del Reino Unido, como lo ha demostrado en la última década la emisión de la importante "Declaración de Cambridge"32.

En efecto, en la creación de los actuales parámetros por los que se rige el Bienestar Animal en Europa, el Reino Unido ha desempeñado un papel muy relevante. En los años 60, la publicación del libro de Ruth Harrison, “Animal Machines"33, tuvo un impacto inmediato en la sociedad y alertó sobre las precarias condiciones de vida de los animales de granja, en régimen de ganadería intensiva.El libro fue un revulsivo y la reacción social que generó determinó que el propio Gobierno británico ordenara la constitución de una Comisión científica, con la misión de elaborar un informe técnico sobre las condiciones de vida de los animales de granja. Como resultado, se publicó el informe presentado en 1965 por el Prof. Roger Brambell, conocido como "The Brambell Report"34, en el que se sustanciaba en qué consistía el Bienestar Animal en cinco mínimos o estándares de vida, para asegurar no sólo la integridad física de los animales, sino incluso la integridad mental, así como el respeto por su propia idiosincrasia, sus modos de vida y de comportamiento acordes con su naturaleza animal. A partir de esa fecha, se puede decir que el tratamiento de los animales y la defensa de sus intereses y el respeto por su comportamiento

\footnotetext{
${ }^{30}$ FRASER, D., Understanding Animal Welfare. The Science in its cultural context (Oxford 2008).

31 VILLALBA, T., 40 años de Bienestar Animal. Guía de la legislación comunitaria sobre Bienestar Animal (Madrid 2016).

32 Vid. The Cambridge Declaration on Consciousness, de 7 de Julio de 2012, en http: / / fcmconference.org/img/CambridgeDeclarationOnConsciousness.pdf

${ }^{33}$ HARRISON R., Animal Machines: The new Factory Farming Industry. Forword by Rachel Carsson (London 1964).

${ }^{34}$ BRAMBELL, R., "Report of the Technical Committee to Enquire into the Welfare of Animals Kept under Intensive Livestock Husbandry Systems”, Her Majesty's Stationery Office (London 1965).
} 
(su "cultura"), ha impregnado la visión académica y las políticas públicas en favor de los animales, con un giro que no ha tenido vuelta atrás.

De hecho, como consecuencia del "Brambell Report", el Gobierno británico creó en 1965 el Farm Animal Welfare Advisory Committee, transformado en 1979 en el "Farm Animal Welfare Commitee” ${ }^{35}$, como órgano responsable del cumplimiento y desarrollo de las políticas de Bienestar Animal, sustanciadas en los cinco principios que constituyen los estándares de Bienestar Animal y que se conocen, como hemos dicho, con el nombre de las Cinco Libertades. ${ }^{36}$

La etapa siguiente (por cierto, aún no clausurada), ha traído consigo la fijación del término "sentient beings", como referente aceptado de forma explícita por el Derecho, para indicar unos mínimos de bienestar cuya regulación, aplicación, respeto y sanción

-en caso de incumplimiento-, incumbe principalmente a los Estados. Es decir, el concepto de Bienestar Animal sería el resultado de la aplicación de la sentiencia animal como parámetro regulador de una vida ( $\mathrm{y}$ una muerte) digna para los animales, lo que se entiende como una responsabilidad pública.

Esto es lo que recogen, de forma sucinta, todos los textos legales, desde que tal término empezó a usarse, en las disposiciones programáticas de Tratados, Protocolos y así mismo, en la aplicación de las políticas públicas de Bienestar Animal en las que particularmente la UE ha desempeñado un papel decisivo ${ }^{37}$. De hecho, ya en 1974 una Directiva del Consejo de Europa, recomendó evitar el sufrimiento innecesario (“unnecessary suffering”) de los animales en el momento del sacrificio ${ }^{38}$. Por lo tanto, la primera trasposición legal de la sentiencia animal consistió, no en una disposición de carácter obligatorio (que los animales no sufran cuando se les sacrifica), sino en la admisión de que sufren y, por tanto, hay que evitarles el sufrimiento nonecesario.

\footnotetext{
${ }^{35}$ Vid. en https://www.gov.uk/government/groups/farm-animal-welfare-committee-fawc

${ }^{36}$ Formuladas originalmente del siguiente modo, Five Freedoms: 1.Freedom from Hunger and Thirst: by ready access to fresh water and a diet to maintain full health and vigor. 2. Freedom from Discomfort: by providing an appropriate environment including shelter and a comfortable resting area. 3. Freedom from Pain, Injury or Disease: by prevention or rapid diagnosis and treatment. 4. Freedom to Express Normal Behavior: by providing sufficient space, proper facilities and company of the animal's own kind. 5 . Freedom from Fear and Distress: by ensuring conditions and treatment which avoid mental suffering.
A. -Lakestani,
N., Animal
Welfare
in
Europe

$37 \quad$ Gavinelli,

(http://www.derechoanimal.info/esp/page/1588/animal-welfare-in-europe)

${ }^{38}$ Council Directive 74/577/EEC of 18 November 1974 on stunning of animals before slaughter, Official Journal $\mathrm{L} \quad 316,26$ November.1974, p. 10-11 (http://eur-lex.europa.eu/legalcontent/EN/TXT/?uri=CELEX\%3A31974L0577)
} 
La Directiva a la que nos referimos no establece sanciones por inferir sufrimiento a los animales (lo que técnicamente no sería posible), sino por exceder el límite de sufrimiento (no se dice dónde está dicho límite) en el momento del sacrificio del animal. Si bien es cierto que una Directiva no puede imponer sanciones por incumplimiento de lo dispuesto en ella, resulta interesante observar que el lenguaje legal ha sido sumamente cauto a la hora de proteger eficazmente a los animales frente al sufrimiento. De hecho, respecto de algunos animales el Derecho emplea el término "maltrato" y éste se sanciona debidamente, mientras que respecto de otros animales, se emplea el término "sufrimiento innecesario". Parece haber, pues, grandes lagunas en la protección animal, que no se cubren solamente con la afirmación en los textos de que los animales son "seres vivos dotados de sensibilidad" ("sentient beings"), como han dispuesto las últimas modificaciones de los Códigos civiles de Francia y de Portugal, para cambiar el estatuto de los animales como cosas en propiedad.

\subsection{La aplicación normativa}

Desde el punto de vista normativo, se podría esperar de la aplicación de la sentiencia de los animales, una mejora de la legislación tanto constitucional, civil, como administrativa y penal muy significativa. Son varios los textos programáticos de la UE que vienen, desde hace 40 años, reconociendo la sentiencia animal como guía de la legislación de Bienestar animal ${ }^{39}$, pero no ha sido hasta el 2009, en el art. 13 del TFUE, llamado Tratado de Lisboa, donde se impone a los Estados Miembros la obligación de tratar a los animales como "seres sintientes" en la legislación interna de cada uno de los Estados Miembros. Este artículo, pues, no excluye a España y tiene carácter vinculante para el Estado.

En dicho artículo 13, se dice de forma taxativa que los animales son "seres sentientes" lo que les aleja de la consideración de cosas en propiedad ${ }^{40}$.

El referido art. 13 dice así en su tenor literal:

Al formular y aplicar las políticas de la Unión en materia de agricultura, pesca, transporte, mercado interior, investigación y desarollo tecnológico y espacio, la Unión y los Estados Miembros tendrán plenamente en cuenta las exigencias en materia de bienestar de los animales como seres sensibles, respetando al mismo tiempo las disposiciones legales o administrativas y las costumbres de los Estados

\footnotetext{
${ }^{39}$ BROOM, D.M., Sentience and Animal Welfare (Boston 2014)136ss.

${ }^{40}$ ALONSO, E., El art.13 del Tratado de Funcionamiento de la Unión Europea, en FAVRE, D. y GIMÉNEZCANDELA, T. (Ed.), Animales y Derecho (Valencia 2015) 18ss.
} 
Miembros relativas, en particular, a ritos religiosos, tradiciones culturales y patrimonio regional ${ }^{41}$.

La claridad con que se expresa el art. 13 respecto a la nueva condición de los animales dentro del ámbito europeo ${ }^{42}$, no ha pasado inadvertida a la doctrina de todo el mundo. En este sentido, conviene poner de relieve, que la UE ha hecho una apuesta decidida por la consideración de los animales como seres sentientes en su legislación sobre Bienestar Animal ya desde 1997 en el tratado de Amsterdam ${ }^{43}$, que es el primer texto legal donde aparece, de forma explícita el empleo del término "sentient beings", referido a los animales.

Un ejemplo claro de las políticas de la UE, dictadas bajo el paradigma de la sentiencia animal, es la Directiva 2003/15/CE ${ }^{44}$, referente a la experimentación animal, que tuvo su reflejo en la Directiva $76 / 768 / \mathrm{CEE}^{45}$, por la que se prohibe la experimentación con animales para productos cosméticos ("testing ban"). Esta normativa europea, que, desde 2009, ha supuesto la prohibición de la venta de productos cosméticos testados en animales, ha sido aplicada en España 10 años más tarde, por medio del Real Decreto 53/2013 de 1 de febrero ${ }^{46}$. Lo interesante es señalar que, tanto en la parte programática de las normas citadas, como en el texto normativo se menciona a los animales como seres sentientes y se señala, como umbral de dolor en los experimentos, el que produce el pinchazo de una inyección.

Valga este texto, aunque hay muchos más, como ejemplo de las modificaciones que cabría esperar de una adecuación de nuestras normas internas relativas a los animales -y particularmente las contenidas en el Código civil-, a su condición de "seres vivos dotados de sensibilidad".

Sin embargo, si la afirmación de la sentiencia animal se queda en una declaración negativa de que los animales "no-son-cosas", no habremos avanzado mucho, pues así lo demuestran los ejemplos de países como Austria, Alemania y Suiza, donde la 'afirmación negativa' -valga la paradoja!- de que los animales "no-son-cosas" (="nicht Sachen") ha llevado a la ineficacia de las medidas que se pusieron en vigor y a la declaración de los jueces de que la

\footnotetext{
${ }^{41}$ Vid. publicación en el BOE del art. 13 TFUE. (https://www.boe.es/buscar/doc.php?id=DOUE-Z-201070006)

${ }^{42}$ WARTEMBERG, M., Art. 13 Lisbon Treaty/TFUE - Historical, Constitutional and Legal Aspects, en FAVRE, D. y GIMÉNEZ-CANDELA, T. (Ed.), Animales y Derecho (Valencia 2015) 353ss.

${ }^{43}$ Treaty of Amsterdam (http://www.europarl.europa.eu/topics/treaty/pdf/amst-en.pdf).

${ }^{44}$ Directiva 2003/15/CE (http://www.derechoanimal.info/bbdd/Documentos/84.pdf).

${ }^{45}$ Directiva76/768/CEE (http://eur-lex.europa.eu/legal-content/ES/TXT/?uri=URISERV\%3Al21191)

${ }^{46}$ RD 53/2013 de 1 de febrero (http://www.derechoanimal.info/bbdd/Documentos/1029.pdf)
} 
negación de que los animales son cosas resulta inaplicable, por ejemplo, a la hora de impedir un embargo sobre un animal de compañía.

En otros términos. La afirmación de la sentiencia animal en los textos legales debe de ir más allá de una afirmación de maquillaje, para servir de freno a la realidad de los abusos que se comenten impunemente contra los animales, y debe de ser consecuente con la responsabilidad que, tanto a los ciudadanos de a pie, como a los Estados les incumbe para velar por un efectivo respeto por los intereses de los animales. He dicho intereses, algunos de ellos tan primarios y evidentes como vivir una vida digna de ser vivida y no morir para divertir a otros.

\subsection{Qué significa Descosificación de los animales}

Dicho de forma sumaria, descosificar es proteger eficazmente, no teorizar sobre la naturaleza del sufrimiento de los animales o establecer cautelarmente las fronteras del "sufrimiento innecesario".

Descosificar es dar un trato igual a todos los animales, lo que no significa la atribución de los mismos derechos subjetivos que a los seres humanos, pero sí significa otorgar a los animales el mismo nivel de exigencia en la protección de sus intereses que damos a los seres humanos.

Descosificar es educar en el respeto a todos los animales, incluso a los que declaramos “plagas" ${ }^{47}$ y a aquellos que, de momento, quedan fuera de toda protección efectiva como son los animales pertenecientes a la Fauna silvestre (o salvaje).

Por ello, la tarea de Descosificación de los animales no es banal ni circunstancial. Es una tarea que apela a la responsabilidad del Estado, a la responsabilidad de los ciudadanos, a la responsabilidad de las Administraciones públicas, a la responsabilidad de las Fuerzas de Seguridad.

\section{REFERÉNCIAS}

ABGB §285a, de 1 de Julio de 1988 (BGBL 179/188; JGS Nr.946/1811):“ § 285a Tiere sind keine Sachen; sie werden durch besondere Gesetze geschützt. Die für Sachen geltenden Vorschriften

${ }^{47}$ GIMÉNEZ-CANDELA,T. - SOUTHER, C., Invasive Animal Species: International Impacts and Inadequate Interventions, en ABATE, R. (Ed.) What can Animal Law learn from Enviromental Law? (Washington DC 2015). 
sind auf Tiere nur insoweit anzuwenden, als keine abweichenden Regelungen bestehen. (=Animals are not things; they are protected by special statutes. Provisions applicable to things only apply to animals to the extent there are no different provisions).

Alemania: BGB $\$ 90^{a}$, de 20 de Agosto de 1990, BGBI I, p.1762); Suiza: ZGB, modificación de 4 de Octubre de 2002 (AS 2003, 463; BBI 2002 4164, 5806); Liechtenstein: Art. 20a Liechtensteinisches Sachenrecht, de 14 de Mayo de 2003, sobre el cambio del Derecho de Propiedad, LR 214.0, 2003 N. 155)

ALONSO, E., El art.13 del Tratado de Funcionamiento de la Unión Europea, en FAVRE, D. y GIMÉNEZ-CANDELA, T. (Ed.), Animales y Derecho (Valencia 2015) 18ss.

ARAUJO, F., A hora dos Direitos dos Animais. O Obstaculo da Apropriaçao (Coimbra 2003) 303ss.; GIMÉNEZ-CANDELA, T., Reforma del Cc. de Portugal: los animales como seres sintientes (http://www.derechoanimal.info/esp/page/4990/reforma-del-cc-de-portugal-los-animalescomo-seres-sintientes)

BOISSEAU-SOWINSKI, L., La désappropiation de l'animal (Presses Universitaires de Limoges 2013).

BRAMBELL, R., "Report of the Technical Committee to Enquire into the Welfare of Animals Kept under Intensive Livestock Husbandry Systems", Her Majesty's Stationery Office (London 1965).

BROOM, D.M., Sentience and Animal Welfare (Boston 2014)136ss.

Codina, Jl., Unanimidad en el Congreso de los Diputados para instar la reforma del Código civil Español y reconocer a los animales como seres dotados de sensibilidad (http://www.derechoanimal.info/images/pdf/SeresDotadosSensibilidad.pdf

Council Directive 74/577/EEC of 18 November 1974 on stunning of animals before slaughter, Official Journal L 316, 26 November.1974, p. 10-11 (http://eur-lex.europa.eu/legalcontent/EN/TXT/?uri=CELEX\%3A31974L0577)

Diario de Sesiones del Congreso de los Diputados Pleno y Diputación Permanente, 14.2.2017 (29) 43ss. (http://www.congreso.es/public_oficiales/L12/CONG/DS/PL/DSCD-12-PL-29.PDF)

DIERAUER, U., Tier und Mensch im Denken der Antike (Amsterdam 1977); INGVILD GILHUS, A., Animals, Gods and Humans (London-New York 2006); ALEXANDRIDIS, WILD, WINKLER-HORACEK (ed.), Mensch und Tier in der Antike (Wiesbaden 2008).

Directiva 2003/15/CE (http://www.derechoanimal.info/bbdd/Documentos/84.pdf).

Directiva76/768/CEE (http://eur-lex.europa.eu/legal-content/ES/TXT/?uri=URISERV\%3Al21191) DUNCAN, I.J.H., The Changing Concept of Animal Sentience, en Applied Animal Behaviour Science 100 (2006) 11ss.

FAVRE, D., Animal Law, Welfare, Interests and Rights (New York 2011); How Common Law Equity Concepts can help enhance Animal's Status, en Animals and the Law (Valencia 2015)181ss. 
FRASER, D., Understanding Animal Welfare. The Science in its cultural context (Oxford 2008). Gavinelli, A.-Lakestani, N., Animal Welfare in Europe

(http://www.derechoanimal.info/esp/page/1588/animal-welfare-in-europe)

GIMÉNEZ-CANDELA, T., An Overview of Spanish Animal Law, en FAVRE, D. y GIMÉNEZ-CANDELA, T. (Ed.), Animales y Derecho (Valencia 2015) 211ss.

GIMÉNEZ-CANDELA, T., Brexit y los animales. El legado del Reino Unido al Derecho Animal (http://www.derechoanimal.info/esp/page/4752/brexit-y-los-animales-el-legado-del-reinounido-al-derecho-animal)

GIMÉNEZ-CANDELA, T., Derecho Privado Romano (Valencia 1999)

GIMÉNEZ-CANDELA, T., La Descosificación de los Animales (I)

(http://www.derechoanimal.info/esp/page/5108/la-descosificacion-de-los-animales-(I))

GIMÉNEZ-CANDELA,T. - SOUTHER, C., Invasive Animal Species: International Impacts and Inadequate Interventions, en ABATE, R. (Ed.) What can Animal Law learn from Enviromental Law? (Washington DC 2015).

Hall, B.-Arellano, S.-Ramírez Barreto, C., En memoria de Tom Regan

(http://www.derechoanimal.info/esp/page/5109/en-memoria-de-tom-regan)

HARRISON R., Animal Machines: The new Factory Farming Industry. Forword by Rachel Carsson (London 1964).

KELCH, T., A Short History of (mostly) Western Animal Law: Part I, Animal Law Review (2012) 1ss.; Part II, Animal Law Review (2013) 1ss.

MARGUÉNAUD, JP., L'entrée en vigueur de “l'amendement Glavany”: un grand pas de plus vers la personnalité juridique des animaux, en RSDA 2 (2014) 15ss.; GIMÉNEZ-CANDELA, T., Una nueva Revolución Francesa: la modernización del Code civil (http://www.derechoanimal.info/esp/page/3718/una-nueva-revolucion-francesa-lamodernizacion-del-code-civil

MUÑOZ MACHADO, S., Los animales y el Derecho (Madrid 1999) 70s.

N. Pedersen, European Animal Welfare Laws: Past, Present and Future, en Animal Law, 2009 (https://www.animallaw.info/intro/eu-us-comparative-cruelty-laws-2003-present) OBERGFELL, E.I., Tiere als Mitgeschöpft im Zivilrecht, en Rechtswissenschaft 3 (2016) 388ss. PETERS, A., Vom Tierschutzrecht zu Legal Animal Studies: Forschungsdesiderate und perspectiven, en 3 (2016)332s.

POLLO, S., Umani e Animali: questioni di Etica (Roma 2016).

RD 53/2013 de 1 de febrero (http://www.derechoanimal.info/bbdd/Documentos/1029.pdf)

REGAN, T., The Case for Animal Rights (University California Press 1983); CAPACETE, F., En recuerdo de Tom Regan(http://www.derechoanimal.info/esp/page/5091/en-recuerdo-de-tomregan) 
ISSN 1981-3694

(DOI): $10.5902 / 1981369426664$

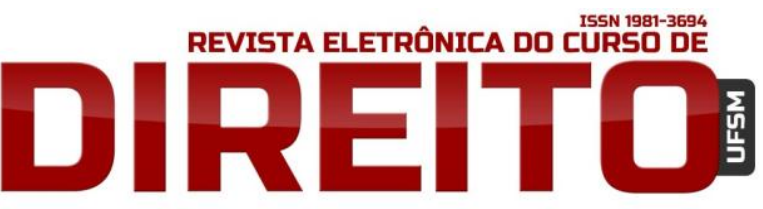

LA DESCOSIFICACIÓN DE LOS ANIMALES

MARITA GIMÉNEZ-CANDELA

RIOS CORBACHO, JM., Comentario en relación al maltrato de animales. LO1/2015

(http://www.derechoanimal.info/images/pdf/Corbacho-Reforma.pdf; REQUEJO CONDE, MC., El delito de maltrato a los animales tras la reforma del Código Penalpor la ley Orgánica 1/2015 de 30 de marzo (http://www.derechoanimal.info/images/pdf/Requejo.pdf)

ROXIN, K., Sinn und Grenzen Staatlicher Strafe, JuS (1996) 377, 383n.20: “...im

Schmerzempfinden der Tiere, dem sich die Rechtsordnung aus einer Art von kreatürlicher Solidarität annimmt".

TFUE, en http://noticias.juridicas.com/base_datos/Anterior/r3-ttce.html The Cambridge Declaration on Consciousness, de 7 de Julio de 2012, en http: / /fcmconference.org/img/CambridgeDeclarationOnConsciousness.pdf

Treaty of Amsterdam (http://www.europarl.europa.eu/topics/treaty/pdf/amst-en.pdf).

ULPIAN D. 1,1,1,3: Ius naturale est, quod natura omnia animalia docuit: nam ius istud non humani generis proprium, sed omnium animalium, quae in terra, quae in mari nascuntur, avium quoque commune est. hinc descendit maris atque feminae coniunctio, quam nos matrimonium appellamus, hinc liberorum procreatio, hinc educatio: videmus etenim cetera quoque animalia, feras etiam istius iuris peritia censeri.

VILLALBA, T., 40 años de Bienestar Animal. Guía de la legislación comunitaria sobre Bienestar Animal (Madrid 2016).

WARTEMBERG, M., Art. 13 Lisbon Treaty/TFUE - Historical, Constitutional and Legal Aspects, en FAVRE, D. y GIMÉNEZ-CANDELA, T. (Ed.), Animales y Derecho (Valencia 2015) 353ss.

WOHLERS, W., Tierschutz durch Strafrecht?. Zur Legitimation tierschutzstrafrechtlicher Normen, en Rechtswissenschaft 3 (2016) 426ss.

Artigo convidado 